\title{
SURFACE WAVES IN HIGHER ORDER VISCO-ELASTIC MEDIA UNDER THE INFLUENCE OF GRAVITY*
}

\author{
Animesh Mukherjee \\ Indian Institute of Mechanics of Continua \\ 201, Maniktala Main Road, Suite No. 42 \\ Calcutta - 700054, West Bengal, India \\ $P$ R. Sengupta \\ Department of Mathematics \\ University of Kalyani \\ Kalyani, West Bengal, India \\ and \\ Lokenath Debnath \\ Department of Mathematics \\ University of Central Florida \\ Orlando, Florida 32816, USA
}

\begin{abstract}
Based upon Biot's [1965] theory of initial stresses of hydrostatic nature produced by the effect of gravity, a study is made of surface waves in higher order visco-elastic media under the influence of gravity. The equation for the wave velocity of Stonely waves in the presence of viscous and gravitational effects is obtained. This is followed by particular cases of surface waves including Rayleigh waves and Love waves in the presence of viscous and gravity effects. In all cases the wave-velocity equations are found to be in perfect agreement with the corresponding classical results when the effects of gravity and viscosity are neglected.
\end{abstract}

Key words: Surface Waves in Elastic Media, Rayleigh Waves, Stonely Waves, Effect of Viscosity and Gravity.

AMS subject classification: $73 \mathrm{D} 20,73 \mathrm{D} 25$.

* Received: December 1989; Revised: April 1990 


\section{INTRODUCTION}

Love (1911) has studied the effects of gravity on various wave problems and shown that the velocity of Rayleigh waves increases significantly due to the gravitational field when the wavelength of the waves is large. Subsequently, Biot (1965) has developed a mathematical theory of initial stresses to investigate the effects of gravity on Rayleigh waves in an incompressible medium assuming that gravity generates an initial stress hydrostatic in nature. Based upon Biot's theory, Sengupta et al (1974-1987) has investigated the effect of gravity on some problems of elastic waves and vibrations, and on the propagation of waves in an elastic layer. The effect of viscosity was not considered in these studies.

The main purpose of this paper is to study surface waves in higher order visco-elastic solid under the influence of gravity. The equation for the wave velocity of Stonely waves in the presence of viscous and gravitational effects is derived. This is followed by particular cases of surface waves including Rayleigh waves and Love waves. It is shown that in all cases the wave-velocity equations are in excellent agreement with the corresponding classical results [Bulen (1965)] when the effects of viscosity and gravity are neglected.

\section{BASIC EQUATIONS OF MOTION IN A VISCO-ELASTIC MEDIUM.}

We consider two homogeneous semi-infinite visco-elastic media, $M_{1}$ and $M_{2}$ in contact with each other $\left(\mathrm{M}_{2}\right.$ being above $\left.\mathrm{M}_{1}\right)$ along a common horizontal plane boundary. We choose the rectangular Cartesian coordinate system with the origin at any point on the plane boundary and the z-axis normal to $M_{1}$. We assume the disturbance is confined to the neighborhood of the common boundary and examine the possibility of a kind of wave traveling in the positive $\mathrm{x}$ direction. We further assume that at any instant of time all the particular in a line parallel to the $y$-axis have equal displacements, that is, all partial derivatives with respect to $y$ vanish.

Introducing the displacement vector $\mathbf{u}=(\mathrm{u}, \mathrm{v}, \mathrm{w})$ at any point $(\mathrm{x}, \mathrm{y}, \mathrm{z})$ at time $\mathrm{t}$, it is convenient to separate the purely dilatational and purely rotational disturbances associated with the components $u$ and $w$ by introducing the two displacement potentials $\phi$ and 
$\psi$ which are functions of $\mathrm{x}, \mathrm{z}$ and $\mathrm{t}$ in the form

$$
\text { (2.1.ab) } \mathrm{u}=\phi_{\mathrm{x}}-\psi_{\mathrm{z}}, \mathrm{w}=\phi_{\mathrm{z}}+\psi_{\mathrm{x}}
$$

From these results, it follows that

$$
\text { (2.2ab) } \quad \nabla^{2} \phi=\mathrm{u}_{\mathrm{x}}+\mathrm{v}_{\mathrm{y}}+\mathrm{w}_{\mathrm{z}} \equiv \Delta, \nabla^{2} \psi=\mathrm{w}_{\mathrm{x}}-\mathrm{u}_{\mathrm{z}}
$$

In standard notations (Bullen [1965]), the component $\mathbf{v}$ is associated with purely distortional waves, and the quantities $\phi, \psi$ and $\mathrm{v}$ are associated with $\mathrm{P}$ waves, SV waves, and SH waves respectively. The dynamical equations of motion for three-dimensional wave problem under the influence of gravity are

$$
\begin{aligned}
& \frac{\partial \sigma_{11}}{\partial x}+\frac{\partial \sigma_{12}}{\partial y}+\frac{\partial \sigma_{13}}{\partial z}+\rho g \frac{\partial w}{\partial x}=\rho \frac{\partial^{2} u}{\partial t^{2}} \\
& \frac{\partial \sigma_{21}}{\partial x}+\frac{\partial \sigma_{22}}{\partial y}+\frac{\partial \sigma_{23}}{\partial z}+\rho g \frac{\partial w}{\partial y}=\rho \frac{\partial^{2} v}{\partial t^{2}} \\
& \frac{\partial \sigma_{31}}{\partial x}+\frac{\partial \sigma_{32}}{\partial y}+\frac{\partial \sigma_{33}}{\partial z}-\rho g\left(\frac{\partial u}{\partial x}+\frac{\partial v}{\partial y}\right)=\rho \frac{\partial^{2} w}{\partial t^{2}}
\end{aligned}
$$

where $\sigma_{\mathrm{ij}}$ are the stress components, $\rho$ is the density of the medium and $g$ is the acceleration due to gravity.

According to Voigt's definition, the stress-strain relations in a higher-order visco-elastic medium are

$$
\sigma_{i j}=\delta_{i j}\left(\sum_{k=0}^{n} \lambda^{k} \frac{\partial^{k}}{\partial t^{k}}\right) \Delta+2\left(\sum_{k=0}^{n} \mu^{k} \frac{\partial^{k}}{\partial t^{k}}\right) e_{i j},
$$

where $\lambda^{0}, \mu^{0}$ and $\lambda^{1}, \mu^{1}, \lambda^{2}, \mu^{2}, \ldots ., \lambda^{\mathrm{n}}, \mu^{\mathrm{n}}$, are respectively Lame's elastic 
constants and the effect of viscosity constants, respectively.

We substitute (2.6) into equations (2.3) - (2.5), and assume that all partial derivatives with respect to $y$ vanish. This leads us to obtain the following dynamical equations of motion of a general visco-elastic solid under the influence of gravity.

$$
\begin{aligned}
& \left\{\sum_{k=0}^{n}\left(\lambda^{k}+\mu^{k}\right) \frac{\partial^{k}}{\partial t^{k}}\right\} \frac{\partial \Delta}{\partial x}+\left\{\sum_{k=0}^{n} \mu^{k} \frac{\partial^{k}}{\partial t^{k}}\right\} \nabla^{2} u+\rho g \frac{\partial w}{\partial x}=\rho \frac{\partial^{2} u}{d t^{2}}, \\
& \left\{\sum_{k=0}^{n} \mu^{k} \frac{\partial^{k}}{\partial t^{k}}\right\} \nabla^{2} v=\rho \frac{\partial^{2} v}{\partial t^{2}}, \\
& \left\{\sum_{k=0}^{n}\left(\lambda^{k}+\mu^{k}\right) \frac{\partial^{k}}{\partial t^{k}}\right\} \frac{\partial \Delta}{\partial z}+\left\{\sum_{k=0}^{n} \mu^{k} \frac{\partial^{k}}{\partial t^{k}}\right\} \nabla^{2} w-\rho g \frac{\partial u}{\partial x}=\rho \frac{\partial^{2} w}{d t^{2}} .
\end{aligned}
$$

Finally, equations (2.7) - (2.9) can be expressed in terms of the displacement potentials $\phi$ and $\psi$ in the form

$$
\left(\sum_{k=0}^{n} \alpha_{j}^{k^{2}} \frac{\partial^{k}}{\partial t^{k}}\right) \nabla^{2} \phi_{j}+g \frac{\partial \psi_{j}}{\partial x}=\frac{\partial^{2} \phi_{j}}{\partial t^{2}},
$$

$$
\left(\sum_{k=0}^{n} \beta_{j}^{k^{2}} \frac{\partial^{k}}{\partial t^{k}}\right) \nabla^{2} \psi_{j}-g \frac{\partial \phi_{j}}{\partial x}=\frac{\partial^{2} \psi_{j}}{\partial t^{2}}
$$

$$
\left(\sum_{\mathrm{k}=0}^{\mathrm{n}} \beta_{j}^{\mathrm{k}^{2}} \frac{\partial^{\mathrm{k}}}{\partial \mathrm{t}^{\mathrm{k}}}\right) \nabla^{2}(\mathrm{v})_{j}=\frac{\partial^{2}(\mathrm{v})_{\mathrm{j}}}{\partial \mathrm{t}^{2}}
$$

where the suffixes $j=1,2$ have been used to designate quantities for the media $M_{1}$ and $\mathrm{M}_{2}$ respectively and

$$
\alpha_{j}^{k^{2}}=\frac{\left(\lambda_{j}^{k}+2 \mu_{j}^{k}\right)}{\rho_{j}} \text { and } \beta_{j}^{k^{2}}=\frac{\mu_{j}^{k}}{\rho_{j}},(k=0,1,2, \ldots, n)
$$




\section{THE SOLUTION OF THE PROBLEM.}

In order to solve (2.10)-(2.12) for the medium $\mathrm{M}_{1}$, we write down the solutions in the form

$$
\begin{aligned}
& \phi_{1}=\mathrm{F}(\mathrm{z}) \exp \{\mathrm{i} \omega(\mathrm{x}-\mathrm{ct})\} \\
& \psi_{1}=\mathrm{G}(\mathrm{z}) \exp \{\mathrm{i} \omega(\mathrm{x}-\mathrm{ct})\} \\
& (\mathrm{v})_{1}=\mathrm{H}(\mathrm{z}) \exp \{\mathrm{i} \omega(\mathrm{x}-\mathrm{ct})\} .
\end{aligned}
$$

Substituting for $\phi_{1}$ and $\psi_{1}$ into the relations (2.10) and (2.11) we obtain

$$
\left(\frac{d^{2}}{d z^{2}}+h_{1}^{2}\right) F+\frac{i g \omega G_{1}}{\sum_{k=0}^{n}(-1)^{k}(i \omega c)^{k} \alpha_{1}^{k^{2}}}=0
$$

$$
\left(\frac{d^{2}}{d z^{2}}+R_{1}^{2}\right) G-\frac{i g \omega F}{\sum_{k=0}^{n}(-1)^{k}(i \omega c)^{k} \beta_{1}^{k^{2}}}=0
$$

where $h_{1}^{2}=\frac{\omega^{2} c^{2}}{\sum_{k=0}^{n}(-1)^{k}(i \omega c)^{k} \alpha_{1}^{k^{2}}}-\omega^{2}$ and $R_{1}^{2}=\frac{\omega^{2} c^{2}}{\sum_{k=0}^{n}(-1)^{k}(i \omega c)^{k} \beta_{1}^{k^{2}}}-\omega^{2}$.

From equations (3.4) - (3.5) we find that $F$ and $G$ satisfy the ordinary differential equation

$$
\left\{\left(\frac{d^{2}}{d z^{2}}+p_{1}^{2} \omega^{2}\right)\left(\frac{d^{2}}{d z^{2}}+q_{1}^{2} \omega^{2}\right)\right\}\{F, G\}=0,
$$

where 
(3.8ab)

$$
p_{1}^{2}+q_{1}^{2}=\frac{\left(h_{1}^{2}+R_{1}^{2}\right)}{\omega^{2}}, p_{1}^{2} q_{1}^{2}=\frac{\left(h_{1}^{2} R_{1}^{2}-m_{1}^{2}\right)}{\omega^{4}}
$$

with

$$
\mathrm{m}_{1}^{2}=\frac{\omega^{2} \mathrm{~g}^{2}}{\left\{\sum_{\mathrm{k}=0}^{\mathrm{n}}(-1)^{\mathrm{k}} \alpha_{1}^{\mathrm{k}^{2}}(i \omega c)^{\mathrm{k}}\right\}\left\{\sum_{\mathrm{k}=0}^{\mathrm{n}}(-1)^{\mathrm{k}}(i \omega c)^{\mathrm{k}} \beta_{1}^{\mathrm{k}^{2}}\right\}} .
$$

A solution for $\mathrm{F}$ from the equation (3.7) is

$$
\begin{aligned}
F & =A_{1} \exp \left(i \omega p_{1} z\right)+B_{1} \exp \left(i \omega q_{1} z\right)+L_{1} \exp \left(-i \omega p_{1} z\right) \\
& +N_{1} \exp \left(-i \omega q_{1} z\right)
\end{aligned}
$$

where $A_{1}, B_{1}, L_{1}$, and $N_{1}$ are constants.

For surface wave solutions, $\mathrm{F}$ tends to zero at large distances from the boundary. This requirement is fulfilled provided the real part of the argument of the exponential function is negative. In view of this condition, the constants $L_{1}$ and $N_{1}$ in the solution (3.10) for $F$ must vanish in the lower medium $M_{1}$. Then the solution for $\phi_{1}$ in $M_{1}$ is

$$
\phi_{1}=\left\{A_{1} \exp \left(i \omega p_{1} z\right)+B_{1} \exp \left(i \omega q_{1} z\right)\right\} \exp \{i \omega(x-c t)\}
$$

Similarly, we can find the solution for $\psi_{1}$ and $\mathrm{v}_{1}$

$$
\begin{aligned}
& \Psi_{1}=\left\{C_{1} \exp \left(i \omega p_{1} z\right)+D_{1} \exp \left(i \omega q_{1} z\right)\right\} \exp \{i \omega(x-c t)\} \\
& (v)_{1}=\exp \left\{i \omega\left(s_{1} z+x-c t\right)\right\}
\end{aligned}
$$

where

$$
s_{1}=\left\{\rho_{1} \frac{c^{2}}{\sum_{k=0}^{n}(-1)^{k}(i \omega c)^{k} \mu_{1}^{k}}-1\right\}^{1 / 2}
$$


with a positive imaginary part, and $C_{1}$ and $D_{1}$ are constants.

It then follows from (3.4) that $C_{1}$ and $D_{1}$ are related to $A_{1}$ and $B_{1}$ through $C_{1}=n_{1} A_{1}$ and $D_{1}=r_{1} B_{1}$ where

$$
\begin{aligned}
& \mathrm{n}_{1}=\left(\omega^{2} \mathrm{p}_{1}^{2}-\mathrm{h}_{1}^{2}\right) \frac{\sum_{\mathrm{k}=0}^{\mathrm{n}}(-1)^{\mathrm{k}}(i \omega c)^{\mathrm{k}} \alpha_{1}^{\mathbf{k}^{2}}}{i \omega g}, \\
& \mathrm{r}_{1}=\left(\omega^{2} \mathrm{q}_{1}^{2}-\mathrm{h}_{1}^{2}\right) \frac{\sum_{\mathrm{k}=0}^{\mathrm{n}}(-1)^{\mathrm{k}}(i \omega c)^{\mathrm{k}} \alpha_{1}^{\mathrm{k}^{2}}}{i \omega \mathrm{g}} .
\end{aligned}
$$

A similar argument enables us to find the solutions in the upper medium $M_{2}$.

We next formulate the two boundary conditions which must be satisfied for the present problem:

I. The components of displacement at the boundary surface between the media $M_{1}$ and $\mathrm{M}_{2}$ must be continuous at all points and times.

II. The stress components $\sigma_{31}, \sigma_{32}$, and $\sigma_{33}$ must also be continuous at all points and times across the boundary surface.

Using the boundary condition I, from the values of $\phi$ and $\psi$ in the two media, after use of the relation (2.1ab) in each case, we obtain

$$
\begin{aligned}
& A_{1}\left(1-n_{1} p_{1}\right)+B_{1}\left(1-r_{1} q_{1}\right)=A_{2}\left(1+n_{2} p_{2}\right)+B_{2}\left(1+r_{2} q_{2}\right), \\
& E_{1}=E_{2} \text {, and } \\
& A_{1}\left(p_{1}+n_{1}\right)+B_{1}\left(q_{1}+r_{1}\right)=A_{2}\left(-p_{2}+n_{2}\right)+B_{2}\left(-q_{2}+r_{2}\right)
\end{aligned}
$$

The stress components in the visco-elastic media of Voigt's type are given by 


$$
\begin{aligned}
& \left(\sigma_{31}\right)_{j}=\left(\sum_{k=0}^{n} \mu_{j}^{k} \frac{\partial^{k}}{\partial t^{k}}\right)\left(2 \frac{\partial^{2} \phi_{j}}{\partial x \partial z}+\frac{\partial^{2} \Psi_{j}}{\partial x^{2}}-\frac{\partial^{2} \psi_{j}}{\partial z^{2}}\right), \\
& \left(\sigma_{32}\right)_{j}=\left(\sum_{k=0}^{n} \mu_{j}^{k} \frac{\partial^{k}}{\partial t^{k}}\right) \frac{\partial(v)_{j}}{\partial z}, \text { and } \\
& \left(\sigma_{33}\right)_{j}=\left(\sum_{k=0}^{n} \lambda_{j}^{k} \frac{\partial^{k}}{\partial t^{k}}\right) \nabla^{2} \phi_{j}+2\left(\sum_{k=0}^{n} \mu_{j}^{k} \frac{\partial^{k}}{\partial z^{2}}\right)\left(\frac{\partial^{2} \phi_{j}}{\partial z^{2}}+\frac{\partial^{2} \psi_{j}}{\partial x \partial z}\right),
\end{aligned}
$$

where, as before $\mathrm{j}=1,2$ for the media $\mathrm{M}_{1}, \mathrm{M}_{2}$.

Applying the second boundary condition to equation (3.19) - (3.21), we obtain

$$
\begin{aligned}
& \mu_{1}^{*}\left\{A_{1}\left(n_{1} p_{1}^{2}-2 p_{1}-n_{1}\right)+B_{1}\left(r_{1} q_{1}^{2}-2 q_{1}-r_{1}\right)\right\} \\
= & \mu_{2}^{*}\left\{A_{2}\left(n_{2} p_{2}^{2}+2 p_{2}-n_{2}\right)+B_{2}\left(r^{2} q_{2}^{2}+2 q_{2}-r_{2}\right)\right\}, \\
& s_{1} \mu_{1}^{*} E_{1}=-s_{2} \mu_{2}^{*} E_{2}, \\
& A_{1}\left\{\lambda_{1}^{*}\left(1+p_{1}^{2}\right)+2 \mu_{1}^{*} p_{1}\left(p_{1}+n_{1}\right)\right\}+B_{1}\left\{\lambda_{1}^{*}\left(1+q_{1}^{2}\right)+2 \mu_{1}^{*} q_{1}\left(q_{1}+r_{1}\right)\right\} \\
= & A_{2}\left\{\lambda_{2}^{*}\left(1+p_{2}^{2}\right)+2 \mu_{2}^{*} p_{2}\left(p_{2}-n_{2}\right)\right\}+B_{2}\left\{\lambda_{2}^{*}\left(1+q_{2}^{2}\right)+2 \mu_{2}^{*} q_{2}\left(q_{2}-r_{2}\right)\right\}
\end{aligned}
$$

where the asterisks indicate the complex quantities as

$$
\theta^{*}=\theta^{0}+\sum_{\mathrm{k}=0}^{\mathrm{n}}(-1)^{\mathrm{k}}(\mathrm{i} \omega c)^{\mathrm{k}} \theta^{\mathrm{k}}
$$

It follows from equations (3.17) and (3.23) that both $E_{1}$ and $E_{2}$ vanish and hence there is no displacement in the $y$ direction, that is, the is no transverse component of displacement. Thus no $\mathrm{SH}$ waves occur in this case.

By eliminating the constants $A_{1}, B_{1}, A_{2}$, and $B_{2}$ from equations (3.16), (3.18), 
(3.22), and (3.24), we obtain the equation for the wave velocity in determinant form

$$
\text { (3.26) }\left[\begin{array}{cccc}
1-n_{1} p_{1} & 1-r_{1} q_{1} & -\left(1+n_{2} p_{2}\right) & -\left(1+r_{1} q_{2}\right) \\
p_{1}+n_{1} & q_{1}+r_{1} & p_{2}-r_{2} & q_{2}-r_{2} \\
F_{1}\left(p_{1}, n_{1}\right) & F_{1}\left(q_{1}, n_{1}\right) & F_{2}\left(p_{2}, n_{2}\right) & F_{2}\left(q_{2}, r_{2}\right) \\
H_{1}\left(p_{1}, n_{1}\right) & H_{1}\left(q_{1}, n_{1}\right) & H_{2}\left(p_{2}, n_{2}\right) & H_{2}\left(q_{2}, p_{2}\right)
\end{array}\right]=0 \text {, }
$$

where

$$
\begin{aligned}
& \text { (3.27) } \mathrm{F}_{1}(\mathrm{p}, \mathrm{n})=\mu_{1}^{*}\left(\mathrm{p}^{2} \mathrm{n}-2 \mathrm{p}-\mathrm{n}\right), \mathrm{F}_{2}(\mathrm{p}, \mathrm{n})=\mu_{2}^{*}\left(\mathrm{n}-\mathrm{np} \mathrm{p}^{2}-2 \mathrm{p}\right) \\
& \text { (3.28) } \mathrm{H}_{1}(\mathrm{p}, \mathrm{n})=-\lambda_{1}^{*}\left(1+\mathrm{p}^{2}\right)-2 \mu_{1}^{*} \mathrm{p}(\mathrm{p}+\mathrm{n}), \mathrm{H}_{2}=\lambda_{2}^{*}\left(1+\mathrm{p}^{2}\right)-2 \mu_{2}^{*} \mathrm{p}(\mathrm{n}-\mathrm{p}) .
\end{aligned}
$$

The roots of equation (3.26) determine the wave velocity of surface wave propagation along the common boundary between two visco-elastic solid media of the Voigt type in the presence of a gravitational field. In other words, this equation gives the wave velocity of Stonely waves in the presence of viscous and gravity effects. In the absence of these effects, equation (3.8) reduces to that for the classical Stonely waves (Stonely, 1924). Finally, we can derive results for Rayleigh waves and Love waves as special cases of this analysis.

\section{RAYLEIGH WAVES.}

In this case, the upper medium $\mathrm{M}_{2}$ is replaced by vacuum so that the plane boundary now becomes a free surface of the lower medium $\mathrm{M}_{1}$. Consequently, $A_{2}=B_{2}=0$ in equations (3.22) and (3.24), and these equations assume the form

$$
\begin{gathered}
A_{1}\left(n_{1} p_{1}^{2}-2 p_{1}-n_{1}\right)+B_{1}\left(r_{1} q_{1}^{2}-2 q_{1}-r\right)=0 \\
A_{1}\left\{\lambda_{1}^{*}\left(1+p_{1}^{2}\right)+2 \mu_{1}^{*} p_{1}\left(p_{1}+n_{1}\right)\right\}+B_{1}\left\{\lambda_{1}^{*}\left(1+q_{1}^{2}\right)+2 \mu_{1}^{*} q_{1}\left(q_{1}+r_{1}\right)\right\}=0
\end{gathered}
$$


Elimination of the constants $A_{1}$ and $B_{1}$ from equations (4.1) and (4.2) yields the following result:

$$
\begin{aligned}
& \left(\mathrm{n}_{1} \mathrm{p}_{1}^{2}-2 \mathrm{p}_{1}-\mathrm{n}_{1}\right)\left\{\left(1+\mathrm{q}_{1}^{2}\right) \lambda_{1}^{*}+2 \mu_{1}^{*} \mathrm{q}_{1}\left(\mathrm{q}_{1}+\mathrm{r}_{1}\right)\right\} \\
& -\left(\mathrm{r}_{1} \mathrm{q}_{1}^{2}-2 \mathrm{q}_{1}-\mathrm{r}_{1}\right)\left\{\left(1+\mathrm{p}_{1}^{2}\right) \lambda_{1}^{*}+2 \mu_{1}^{*} \mathrm{p}_{1}\left(\mathrm{p}_{1}+\mathrm{n}_{1}\right)\right\}=0 .
\end{aligned}
$$

This is the required wave velocity equation of Rayleigh waves in a higher order visco-elastic solid medium under the influence of gravity. When the effects of gravity and viscosity are ignored, this equation (4.3) reduces to the corresponding classical result for the Rayleigh waves (Bullen (1965)).

\section{LOVE WAVES.}

For the existence of Love waves, we consider a layered semi-infinite medium in which $\mathrm{M}_{2}$ is bounded by two horizontal plane surfaces at a finite distance $\mathrm{H}$ apart, while the lower medium $\mathrm{M}_{1}$ remains infinite as before. We now have to determine only the displacement component $\mathrm{v}$ in the direction of the $\mathrm{y}$-axis.

For the medium $M_{1}$ we proceed exactly as in the general case, and thus $\left(v_{1}\right)$ is given by (3.1) with the imaginary part of $s_{1}$ positive. However, for the medium $\mathrm{M}_{2}$ we must retain the full solution since the displacement no longer diminishes with increasing distance from the common boundary of the two media. Consequently, we have

$$
(v)_{2}=A^{\prime} \exp \left\{i \omega\left(s_{2} z+x-c t\right)\right\}-B^{\prime} \exp \left\{i \omega\left(s_{2} z+x+c t\right)\right\}
$$

where the imaginary part of the complex quantity $s_{2}$ is now not positive.

Since the displacement component $(v)_{2}$ and stress component $\sigma_{32}$ must be continuous across the plane of contact, we have

$$
(\mathrm{v})_{1}=(\mathrm{v})_{2}, \quad\left(\sigma_{32}\right)_{1}=\left(\sigma_{32}\right)_{2} \text { on } \mathrm{z}=0 \text {. }
$$


It follows from (3.13) and (5.1) combined with (5.2ab) that

$$
\text { (5.3ab) } \quad \mathrm{E}_{1}=\mathrm{A}^{\prime}+\mathrm{B}^{\prime}, \quad \mu_{1}^{*} \mathrm{~s}_{1} \mathrm{E}_{1}=\mu_{2}^{*} \mathrm{~s}_{2}\left(\mathrm{~A}^{\prime}-\mathrm{B}^{\prime}\right) .
$$

Elimination of $E_{1}$ between equations (5.3ab) yields

$$
\mathrm{A}^{\prime}\left(\mathrm{s}_{2} \mu_{2}^{*}-\mathrm{s}_{1} \mu_{1}^{*}\right)=\mathrm{B}^{\prime}\left(\mathrm{s}_{2} \mu_{2}^{*}+\mathrm{s}_{1} \mu_{1}^{*}\right)
$$

Also making use of the boundary condition that there is no stress across the free surface

$$
\left(\sigma_{32}\right)_{2}=0 \text { at } \mathrm{z}=-\mathrm{H} \text {, }
$$

we have from equation (5.1)

$$
A^{\prime} \exp \left(-\omega s_{2} H\right)=B^{\prime} \exp \left(i \omega s_{2} H\right) .
$$

Eliminating $\mathrm{A}^{\prime}$ and $\mathrm{B}^{\prime}$ between equations (5.4) and (5.6), we obtain the result

$$
\mathrm{s}_{2} \mu_{2}^{*} \tan \left(\omega \mathrm{s}_{2} \mathrm{H}\right)+\mathrm{is}_{1} \mu_{1}^{*}=0
$$

This is the required wave velocity for Love waves in a higher order visco-elastic medium under the influence of gravity. It is seen from the equation that Love waves are not affected by the presence of a gravitational field. For perfectly elastic media,

$\mu_{1} \mathrm{k}=\mu_{2} \mathrm{k}=0, \quad(\mathrm{k}=1,2, \ldots, \mathrm{n})$, equation (5.7) reduces to the corresponding classical result (Bullen (1965)).

\section{CLOSING REMARKS.}

The present study reveals that effects of viscosity and gravity are reflected in the wave velocity equations corresponding to the Stonely waves, Rayleigh waves, and Love waves. So the results of this analysis seem to be useful in circumstances where these effects cannot be neglected. Some special cases of this study have been discussed by 
several authors including Sengupta et al (1974 - 1987).

ACKNOWLEDGEMENT: This work was partially supported by the University of Central Florida.

\section{REFERENCES}

[1] Biot, M.A., Mechanics of Incremental Deformation, John Wiley, New York (1965)

[2] Bullen, K.E., An Introduction to the Theory of Seismology, Cambridge University Press (1965).

[3] Love, A.E.H., Some Problems of Geodynamics, Dover Publications, New York (1911).

[4] Stonely, R., Proc. Roy. Soc. London A 106: 416-428 (1924)

[5] Sengupta, P.R and De, S.N., Journ. Acous. Soc. Amer. 55: 919-921 (1974)

[6] Sengupta, P.R. and Acharya, D., Gerlands Beitr. Geophys. 87: 141-146 (1978)

[7] Sengupta, P.R., and Roy, S.K., Gerlands Beitr. Geophys. 92: 435-442 (1983) 


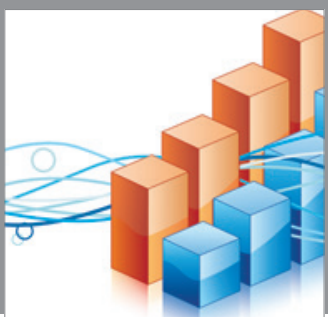

Advances in

Operations Research

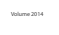

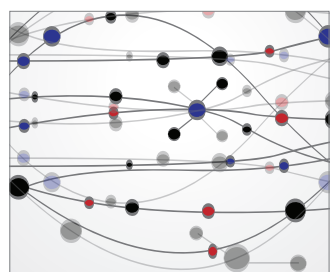

\section{The Scientific} World Journal
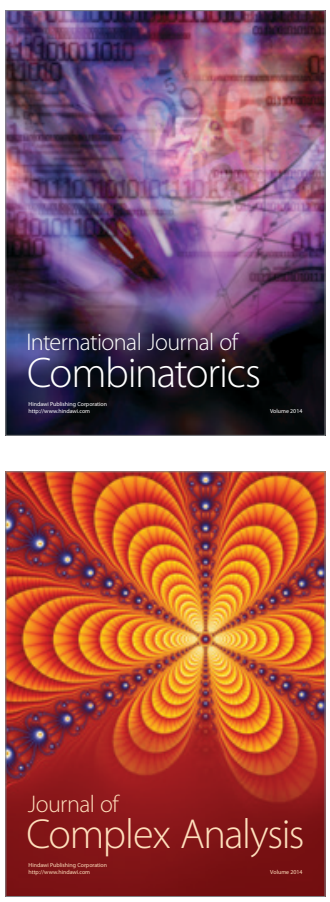

International Journal of

Mathematics and

Mathematical

Sciences
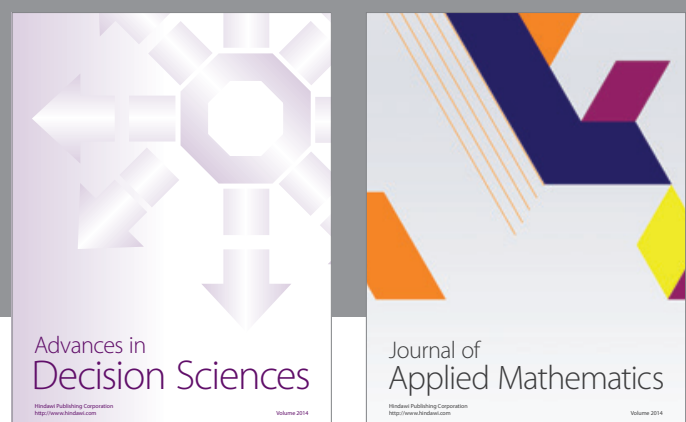

Journal of

Applied Mathematics
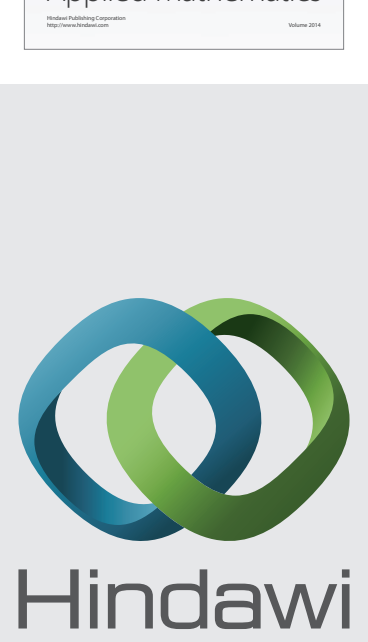

Submit your manuscripts at http://www.hindawi.com
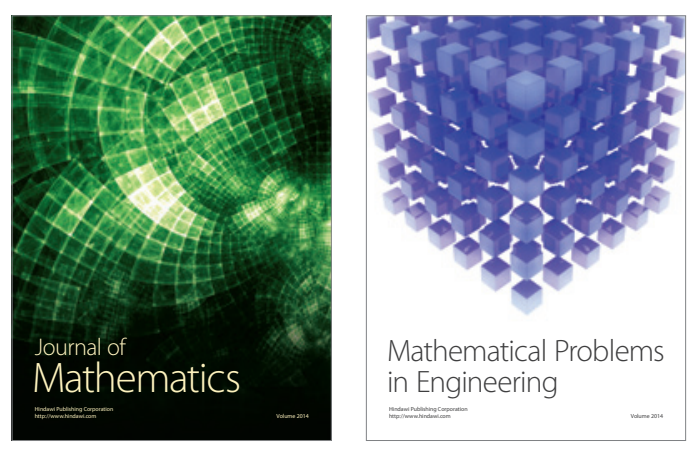

Mathematical Problems in Engineering
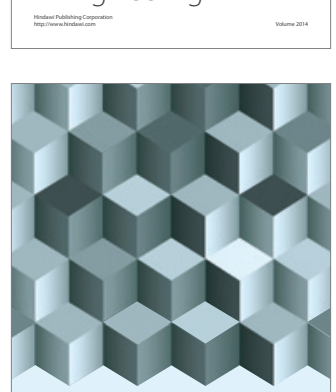

Journal of

Function Spaces
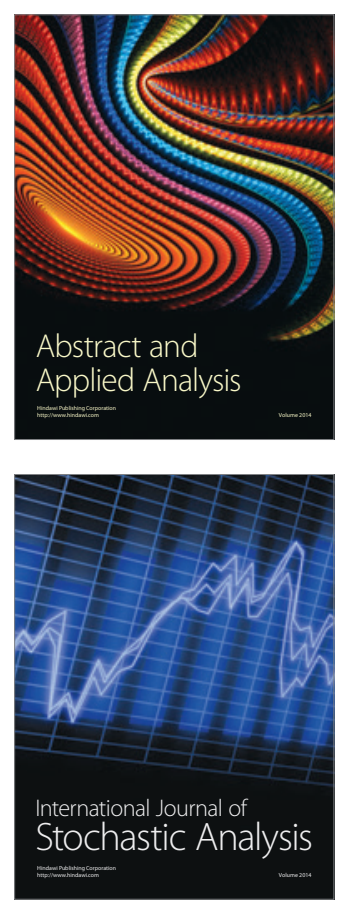

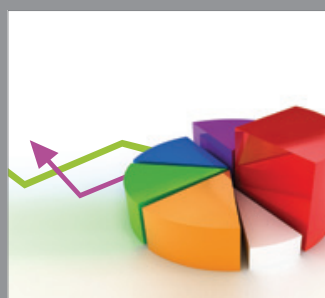

ournal of

Probability and Statistics

Promensencen
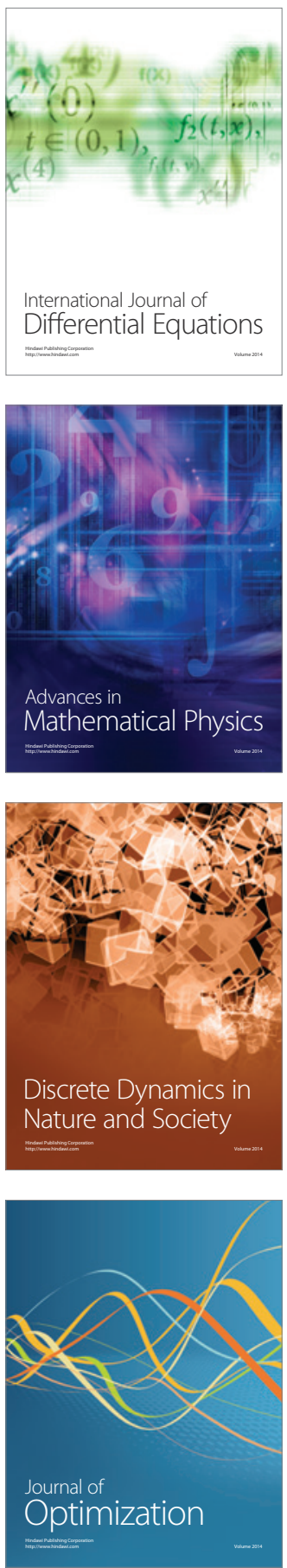